\title{
Geometric Phase Effect at $N$-Fold Electronic Degeneracies in Jahn-Teller Systems
}

\author{
A. J. C. VARANDAS, Z. R. XU* \\ Departamento de Quimica, Universidade de Coimbra, P-3049 Coimbra Codex, Portugal \\ Received 11 December 2002; accepted 10 October 2003 \\ Published online 3 June 2004 in Wiley InterScience (www.interscience.wiley.com). \\ DOI 10.1002/qua.20036
}

\begin{abstract}
The geometric phase effect at arbitrary integral $N$-fold electronic degeneracies is investigated in Jahn-Teller systems. It is shown to be a property inherent to inversion of the adiabatic electronic wave vectors in the $\mathrm{N}$-dimensional electronic wave-vector space. The relationship between the geometric phase and mixing angles has also been established. (C) 2004 Wiley Periodicals, Inc. Int J Quantum Chem 99: 385-392, 2004
\end{abstract}

Key words: geometric phase effect; Jahn-Teller systems; electronic degeneracies; generalized Born-Oppenheimer approximation; conical intersections

\section{Introduction}

A bout four decades have elapsed since Herzberg and Longuet-Higgins [1] showed that a real-valued electronic wave function changes sign when the nuclear coordinates traverse a cyclic path around a conical intersection (two-fold electronic degeneracy; for reviews, see [2-4]). Such a geometric phase (GP) effect was later demonstrated on a wider context by Berry [5], and hence it is also known under his name. Moreover, it is often referred to as the Aharonov-Bohm effect [6] because the differential equations in-

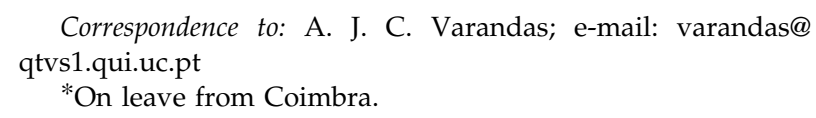

*On leave from Coimbra.

volved are similar to those of a charged particle moving in the presence of a magnetic solenoid. Although research on the GP effect has a long history and is currently a very active field of research [3, 5, 7-21] (references to further work on this topic may be extracted through crossreferencing), the issue of cyclic phases at $N$-fold electronic degeneracy is a much more recent one $[22,23]$. Specifically, for a model Hamiltonian, Manolopoulos and Child [22] studied the possible sign changes that can occur when $N$ real quantum states are transported adiabatically around an $\mathrm{N}$-fold electronic degeneracy, while Baer [23] focused on the topological features and existence of pure diabatic states. The major goal of this work is to examine the case of any $N$-fold electronic degeneracy in Jahn-Teller systems [24, 
25] and to establish the GP angle in terms of the involved mixing angles.

The plan of the paper is as follows. In section 2 we present the theoretical approach. Specifically, we examine in subsections 2.1 and 2.2 the $E \otimes e$ and $T \otimes\left(e+t_{2}\right)$ Jahn-Teller cases, which are prototypes of the $N=2$ and $N=3$ electronic degeneracies, respectively. The method is extended to $N=4$ in subsection 2.3, and generalized to any $N$-fold electronic degeneracy in subsection 2.4. Conclusions are in section 3 .

\section{Theoretical Approach}

In present the theory, we begin with a detailed treatment of the simplest $N=2$ Jahn-Teller system as this is important for an understanding of the higher-index cases. Note that we imply by JahnTeller system the so-called Jahn-Teller center [25], i.e., the group of atoms involved in the degenerate electronic states. Thus, although the orbital doublet of $\mathrm{H}_{3}$ is a well established prototype [4] for the $E \otimes$ $e$ case, the conclusions drawn below are far more general.

\subsection{THE $N=2$ CASE: $E \otimes e$}

As it is well established [25], the degeneracy of electronic states owing to the symmetry of the nuclear configuration (belonging to the point group $G$ ) exists only in polyatomic systems which have at least one rotation $\left(C_{n}\right)$ or roto-reflection $\left(S_{n}\right)$ symmetry axis of order $n>2$. For electronic orbital doublets, there are two types of possible JahnTeller effects. The first occurs when the electronic degeneracy $E_{k}$ is removed by nuclear displacements that transform according to the doublet $E_{2 k}$ representation, the so-called $E \otimes e$ case. The second type is possible only in polyatomic systems with $C_{n}$ or $S_{n}$ axes of order $n=4 k$. In this case, the nuclear displacements of $B_{1}$ or $B_{2}$ symmetry are active in the linear vibronic interaction, leading to the $E \otimes\left(b_{1}+\right.$ $b_{2}$ ) Jahn-Teller case. For the present analysis, we consider only the more widespread $E \otimes e$ case.

The Hamiltonian of the linear $E \otimes e$ Jahn-Teller problem (i.e., quadratic and higher-order terms of the vibronic interaction are ignored), written in the basis of electronic states $\left\{\left|E_{x}(\mathbf{r})\right\rangle,\left|E_{y}(\mathbf{r})\right\rangle\right\}$ where $\mathbf{r}$ is the set of electronic coordinates, assumes the form [25]

$$
\mathbf{H}(\mathbf{Q})=H_{\mathrm{e}}+\mathbf{H}_{\mathrm{v}}+\mathbf{H}_{\mathrm{ev}}
$$

$$
\begin{gathered}
\mathbf{H}_{\mathrm{e}}=E_{0} \boldsymbol{\sigma}_{0} \\
\mathbf{H}_{\mathrm{v}}=\frac{1}{2}\left[P_{E_{x}}^{2}+P_{E_{y}}^{2}+\omega_{E}^{2}\left(Q_{E_{x}}^{2}+Q_{E_{y}}^{2}\right)\right] \boldsymbol{\sigma}_{0} \\
\mathbf{H}_{\mathrm{ev}}=V_{E}\left(Q_{E_{x}} \boldsymbol{\sigma}_{x}+Q_{E_{y}} \boldsymbol{\sigma}_{y}\right),
\end{gathered}
$$

with $Q_{\Gamma \gamma}$ being the $\gamma$ component of the nuclear displacements which transform according to the irreducible representation $\Gamma, P_{\Gamma \gamma}$ the corresponding conjugate momentum, $\mathbf{Q}=\left\{Q_{\Gamma \gamma}\right\}$ the nuclear coordinates, and $\mathbf{Q}_{0}$ a reference nuclear configuration which will be taken as the point of electronic degeneracy. In turn, $\left|E_{x}(\mathbf{r})\right\rangle=\left|E_{x}\left(\mathbf{r}, \mathbf{Q}_{0}\right)\right\rangle$ is one of the eigenvectors of the Hamiltonian $\mathbf{H}\left(\mathbf{Q}_{0}\right)$, and so on. Furthermore, $E_{0}$ is the energy of the orbital doublet, $\omega_{E}$ is the vibrational frequency of the $E$ mode, $V_{E}$ is the so-called linear vibronic interaction constant, and $\boldsymbol{\sigma}_{i}$ are the well-known Pauli matrices.

The symmetry groups of the electronic Hamiltonian $\mathbf{H}_{\mathrm{e}}$ and the vibrational Hamiltonian $\mathbf{H}_{\mathrm{v}}$ are both at least the second-order special unitary group $S U(2)$. Thus, in the absence of a vibronic interaction, the symmetry of the $E \otimes e$ Jahn-Teller system must be described by the Lie group $S U(2) \times S U(2)$. An arbitrary element of this group will then depend on six parameters [25-27], namely

$$
\mathbf{G}=\exp [i(\boldsymbol{\xi} \boldsymbol{\sigma}+\boldsymbol{\alpha} \mathbf{L})],
$$

with the parameters of $\boldsymbol{\xi}=\left\{\xi_{i}\right\}$ and $\boldsymbol{\alpha}=\left\{\alpha_{i}\right\}(i=x$, $y, z)$. The vibronic interaction leads to a lowering of the symmetry of the problem. For example, by taking into account the linear vibronic interaction, the symmetry of the system is known [25] to reduce to the one-parameter $\left(\alpha_{z}\right)$ axial group $O(2)$. The group element then assumes the form

$$
\hat{G}=\exp \left[i \alpha_{z}\left(L_{z}+\frac{1}{2} \boldsymbol{\sigma}_{z}\right)\right],
$$

which is a result of the requirement that $[\hat{G}, \hat{H}]=0$, or

$$
\left[(\boldsymbol{\xi} \boldsymbol{\sigma}+\boldsymbol{\alpha} \mathbf{L}),\left(Q_{E_{x}} \boldsymbol{\sigma}_{x}+Q_{E_{y}} \boldsymbol{\sigma}_{y}\right)\right]=0
$$

In fact, Eq. (7) leads to the following restrictions:

$$
\begin{aligned}
& \alpha_{x}=\alpha_{y}=0, \\
& \xi_{x}=\xi_{y}=0,
\end{aligned}
$$




$$
\alpha_{z}=2 \xi_{z}
$$

and hence reduces the six parameters to just one parameter. As a result, the corresponding infinitesimal operator [24, 25]

$$
\hat{J}_{z}=\hat{L}_{z}+\frac{1}{2} \hat{\sigma}_{z}
$$

will be an integral of motion because it commutes with the Hamiltonian in Eq. (1), with the operator $\hat{L}_{z}$ assuming in polar coordinates $\left(Q_{x}=\rho \cos \phi\right.$, $\left.Q_{y}=\rho \sin \phi\right)$ the form

$$
\hat{L}_{z}=-i \frac{\partial}{\partial \phi}
$$

The Hamiltonian $\mathbf{H}$ can be diagonalized by the unitary transformation [25]

$$
\mathbf{S}^{\dagger}\left(Q_{E_{x}} \boldsymbol{\sigma}_{x}+Q_{E_{y}} \boldsymbol{\sigma}_{y}\right) \mathbf{S}=\rho \boldsymbol{\sigma}_{z}
$$

where $\mathbf{S}$ is a unitary matrix defined by

$$
\mathbf{S}=\frac{1}{\sqrt{2}}\left[\begin{array}{cc}
\exp (-i \phi / 2) & \exp (-i \phi / 2) \\
\exp (i \phi / 2) & -\exp (i \phi / 2)
\end{array}\right]
$$

Such a transformation leads to [25]

$$
\hat{S}^{+} \hat{J}_{z} \hat{S}=\hat{L}_{z}
$$

which shows that $\hat{L}_{z}$ is an integral of motion in the adiabatic representation.

In the absence of a vibronic interaction, the motions of the electronic and nuclear subsystems can be treated separately. The electronic states $\left|E_{x}(\mathbf{r})\right\rangle$ and $\left|E_{y}(\mathbf{r})\right\rangle$, with certain values of the projection of the energy spin $1 / 2 \boldsymbol{\sigma}$ on the $z$ axis, will then correspond (in the case of an $X_{3}$ molecule) to the wave of electronic density propagating along the perimeter of the molecule clockwise or counterclockwise, respectively. In turn, the vibrational states with a certain value of $L_{z}$ correspond to the wave of distortions of an equilateral triangle running clockwise or counterclockwise. The energy spin $1 / 2 \boldsymbol{\sigma}$ and vibrational momentum $\mathbf{L}$ are in such a case conserved separately. This ceases to be the case when the vibronic coupling is introduced. The projection of the vibronic angular momentum $\mathbf{J}=\mathbf{L}+1 / 2 \boldsymbol{\sigma}$ onto the $z$ axis now becomes the good quantum number, with the waves of electronic density and nuclear distortions propagating coherently.

As a result of the above analysis, the adiabatic electronic wave vectors $\left\{\left|\psi_{I}(\mathbf{r} ; \mathbf{Q})\right\rangle\right\}$ propagate around the conical intersection as the one-parameter, two-dimensional (2D) rotation $[O(2)]$ of the initial electronic wave vectors $\left|E_{x}(\mathbf{r})\right\rangle$ and $\left|E_{y}(\mathbf{r})\right\rangle$. We emphasize that the symmetry of the Hamiltonian for the $E \otimes e$ Jahn-Teller system is $O(2)$, not $S O(2)$, because, for arbitrary rotations around the $z$ axis, the Hamiltonian has two extra symmetry operations [28]: $\mathbf{P}=\hat{R}_{E_{y}} \boldsymbol{\sigma}_{x}$, where $\hat{R}_{E_{y}}$ is an operator that changes the sign of $Q_{E_{y^{\prime}}}$ and $\boldsymbol{\Theta}=\hat{K} \boldsymbol{\sigma}_{x}$, where $\hat{K}$ is the complex conjugation operator.

In polar coordinates, the adiabatic electronic wave vectors will then propagate around the conical intersection as

$$
\left(\begin{array}{c}
\left|\psi_{1}\left(\mathbf{r} ; \xi_{z}\right)\right\rangle \\
\left|\psi_{2}\left(\mathbf{r} ; \xi_{z}\right)\right\rangle
\end{array}\right)=\left[\begin{array}{cc}
\cos \xi_{z} & -\sin \xi_{z} \\
\sin \xi_{z} & \cos \xi_{z}
\end{array}\right]\left(\begin{array}{l}
\left|E_{x}(\mathbf{r})\right\rangle \\
\left|E_{y}(\mathbf{r})\right\rangle
\end{array}\right),
$$

where the angle $\xi_{z}=\alpha_{z} / 2$ is the relevant parameter. In matrix notation, one has

$$
\boldsymbol{\psi}\left(\mathbf{r} ; \xi_{z}\right)=\mathbf{R}_{2}\left(\xi_{z}\right) \boldsymbol{\psi}(\mathbf{r})
$$

Clearly, for $\xi_{z} \rightarrow \xi_{z}+\pi$, one has $\mathbf{R}_{2}\left(\xi_{z}+\pi\right)=$ $-\mathbf{R}_{2}\left(\xi_{z}\right)$. This implies that, upon inversion $\left(\hat{\imath}_{2}\right)$, the adiabatic electronic wave vectors change direction in the electronic wave-vector space $\left\{\left|E_{x}(\mathbf{r})\right\rangle,\left|E_{y}(\mathbf{r})\right\rangle\right\}$. We now note that $\alpha_{z}$ is the angle associated to the 2-D nuclear configuration space spanned by $Q_{x}$ and $Q_{y}$. In polar coordinates, it will then be identical to $\phi$, a notation that we keep from here onward. Thus, $\phi=2 \xi_{z}$, which implies that inversion in the 2-D electronic wave-vector space leads to the GP effect [1] (i.e., the wave function changes sign when transported adiabatically along a loop in configuration space that encircles the degeneracy point). To our knowledge, this provides a novel understanding of the GP effect for systems with an orbital doublet degeneracy. We emphasize that the electronically adiabatic wave vectors must reverse their direction under inversion in the electronic wave-vector space or, equivalently, after closing a loop in configuration space around the conical intersection. Because a physical state may be described by any wave vector $|\psi\rangle$ in a ray [27], $\exp [i A(\mathbf{R})]|\psi\rangle$, the corresponding real wave function will be double-valued (in other words, two opposing wave vectors represent a unique physical state). 
Similarly to Eq. (16), the general adiabatic electronic wave vectors $\left\{\left|\psi_{I}(\mathbf{r} ; \mathbf{R})\right\rangle\right\}$ can be represented by a one-parameter orthogonal transformation of the initial electronic wave vectors $\left|E_{x}(\mathbf{r})\right\rangle$ and $\left|E_{y}(\mathbf{r})\right\rangle$; $\mathbf{R}$ represents the full set of nuclear coordinates. For the two-state problem, one has

$$
\left(\begin{array}{c}
\left|\psi_{1}(\mathbf{r} ; \mathbf{R})\right\rangle \\
\left|\psi_{2}(\mathbf{r} ; \mathbf{R})\right\rangle
\end{array}\right)=\left[\begin{array}{cc}
\cos \gamma(\mathbf{R}) & -\sin \gamma(\mathbf{R}) \\
\sin \gamma(\mathbf{R}) & \cos \gamma(\mathbf{R})
\end{array}\right]\left(\begin{array}{l}
\left|E_{x}(\mathbf{r})\right\rangle \\
\left|E_{y}(\mathbf{r})\right\rangle
\end{array}\right),
$$

or, in matrix form,

$$
\boldsymbol{\psi}(\mathbf{r} ; \mathbf{R})=\mathbf{T}_{2}(\gamma(\mathbf{R})) \boldsymbol{\psi}(\mathbf{r}),
$$

where $\gamma(\mathbf{R})$ is the mixing angle [16, 17, 29] (this has been shown [17] to be equivalent, up to a constant, to the so-called adiabatic to diabatic transformation angle [30-33]). We observe that $\psi_{i}(i=1,2)$ are real and that they change sign over a closed loop around the degeneracy point. Clearly, this prevents the use of the standard Born-Oppenheimer treatment for each state since the involved electronic wave functions are not well behaved. To develop a generalized Born-Oppenheimer approach to the problem in the vicinity of the degeneracy seam, we consider the auxiliary electronic wave functions

$$
\begin{aligned}
& \tilde{\psi}_{a}(\mathbf{r} ; \mathbf{R})=\frac{1}{\sqrt{2}}\left(\psi_{1}+i \psi_{2}\right), \\
& \tilde{\psi}_{b}(\mathbf{r} ; \mathbf{R})=\frac{1}{\sqrt{2}}\left(i \psi_{1}+\psi_{2}\right),
\end{aligned}
$$

where the tilde indicates that we are dealing with complex wave functions; whenever causing no ambiguity, we omit the dependence of the wave functions on the electronic and nuclear coordinates. From Eq. (20), we obtain [34]

$$
\left\langle\tilde{\psi}_{a} \mid \nabla \tilde{\psi}_{a}\right\rangle=i\left\langle\psi_{1} \mid \nabla \psi_{2}\right\rangle,
$$

where the bracket notation implies integration over the electronic coordinates. A similar result, except for the sign, holds for $\left\langle\tilde{\psi}_{b} \mid \nabla \tilde{\psi}_{b}\right\rangle$. We will use only $\tilde{\psi}_{a}$ in the following discussion, although the result is later generalized to $\tilde{\psi}_{b}$. Consider now Eq. (20) written in the form

$$
\tilde{\psi}_{a}(\mathbf{r} ; \mathbf{R})=\exp [i B(\mathbf{r}, \mathbf{R})] \psi_{a}(\mathbf{r} ; \mathbf{R}),
$$

where $B(\mathbf{r}, \mathbf{R})$ is a phase which we assume for generality to depend both on the nuclear and electronic coordinates. Using Eq. (23), one obtains

$$
\left\langle\tilde{\psi}_{a}(\mathbf{r} ; \mathbf{R}) \mid \nabla \tilde{\psi}_{a}(\mathbf{r} ; \mathbf{R})\right\rangle=i \nabla A(\mathbf{R})+\left\langle\psi_{a}(\mathbf{r} ; \mathbf{R}) \mid \nabla \psi_{a}(\mathbf{r} ; \mathbf{R})\right\rangle,
$$

where $\nabla A(\mathbf{R})=\left\langle\tilde{\psi}_{a}|\nabla B(\mathbf{r} ; \mathbf{R})| \tilde{\psi}_{a}\right\rangle$. By now comparing Eq. (22) with Eq. (24), one obtains $\nabla A(\mathbf{R})=\left\langle\psi_{1} \mid \nabla \psi_{2}\right\rangle$. Furthermore, by recalling [16, 17] that $\left\langle\psi_{1} \mid \nabla \psi_{2}\right\rangle=$ $\nabla \gamma(\mathbf{R})$, we may write, up to a constant,

$$
A(\mathbf{R})=\gamma(\mathbf{R}) .
$$

Because the mixing angle $\gamma(\mathbf{R})$ has been shown [17, 19] to lead to the proper sign change when looping around a conical intersection (as well as the proper behavior in loops which do not encircle it), we may, for most purposes, identify $A(\mathbf{R})$ with the GP angle; note that $\Delta \gamma=\pi$ for a cyclic path encircling the conical intersection, otherwise $\Delta \gamma=0$. Finally, we return to Eq. (21) to note that a development similar to the one carried out above for $\tilde{\psi}_{a}$ leads to $A(\mathbf{R})=$ $-\gamma(\mathbf{R})$ in the case of $\tilde{\psi}_{b}$. Of course, the sign change has no practical implications, as it simply implies a clockwise measurement of the phase angle if positive phase angles are assumed to be defined counterclockwise in Eq. (25).

\subsection{THE $N=3$ CASE: $T \otimes\left(e+t_{2}\right)$}

We turn next to three-fold electronic degeneracies which are associated to cubic and icosahedral symmetry groups [25]. The doubly degenerate $E$ type vibrations and the triply degenerate $T$-type vibrations are now Jahn-Teller active, leading to the $T \otimes\left(e+t_{2}\right)$ problem of which the best wellknown example is perhaps that of an octahedral $\mathrm{ML}_{6}$ molecule $\left(O_{h}\right.$ point group). Without vibronic interactions, the Jahn-Teller Hamiltonian has in this case Lie group symmetry $S U(3) \times S U(5)$, reducing to $S O(3)$ if the linear vibronic interaction is included [25]. This implies three Lie group parameters. However, of the three $\left\{\left|E_{x}(\mathbf{r})\right\rangle,\left|E_{y}(\mathbf{r})\right\rangle,\left|E_{z}(\mathbf{r})\right\rangle\right\}$ electronic wave vectors, only two are independent as their sum of squares must be equal to unity. Thus, any adiabatic electronic wave vector can be characterized in terms of two angles and a modulus in the $\left\{\left|E_{x}(\mathbf{r})\right\rangle,\left|E_{y}(\mathbf{r})\right\rangle,\left|E_{z}(\mathbf{r})\right\rangle\right\}$ space. This implies that we may choose those two angles from three Lie group parameters: one has three sets of two angles as possible choices. 
Now, by analogy with the $n=2$ case, we assign to each chosen parameter a $3 \times 3$ unitary planar rotation matrix [35]. Thus, the full matrix assumes the form

$$
\mathbf{T}_{3}=\mathbf{t}_{12}\left(\xi_{12}\right) \mathbf{t}_{23}\left(\xi_{23}\right)
$$

where $\xi_{i j}$ are the two relevant angles in the 3-D polar coordinate system, and $\mathbf{t}_{n m}$ are $3 \times 3$ matrices defined by $\left[\mathbf{t}_{n m}\right]_{n n}=\left[\mathbf{t}_{n m}\right]_{m m}=\cos \left(\xi_{n m}\right)$ and $\left[\mathbf{t}_{n m}\right]_{n m}=-\left[\mathbf{t}_{n m}\right]_{m n}=\sin \left(\xi_{n m}\right)$, with all other entries satisfying $\left[\mathbf{t}_{n m}\right]_{i j}=\delta_{i j}$. The real electronic wave functions will now be given by the three rows of $\mathbf{T}_{3}$ matrix, namely

$$
\begin{array}{r}
\left(\begin{array}{c}
\left|\psi_{1}\left(\mathbf{r} ; \xi_{23}, \xi_{12}\right)\right\rangle \\
\left|\psi_{2}\left(\mathbf{r} ; \xi_{23}, \xi_{12}\right)\right\rangle \\
\left|\psi_{3}\left(\mathbf{r} ; \xi_{23}, \xi_{12}\right)\right\rangle
\end{array}\right) \\
=\left(\begin{array}{ccc}
\cos \xi_{23} \sin \xi_{12} & \sin \xi_{23} \sin \xi_{12} & \cos \xi_{12} \\
\cos \xi_{23} \cos \xi_{12} & \sin \xi_{23} \cos \xi_{12} & -\sin \xi_{12} \\
-\sin \xi_{23} & \cos \xi_{23} & 0
\end{array}\right] \\
\times\left(\begin{array}{l}
\left|E_{x}(\mathbf{r})\right\rangle \\
\left|E_{y}(\mathbf{r})\right\rangle \\
\left|E_{z}(\mathbf{r})\right\rangle
\end{array}\right),
\end{array}
$$

which shows that, under inversion $\hat{\imath}_{3}$ in $\left\{\left|E_{x}(\mathbf{r})\right\rangle\right.$, $\left.\left|E_{y}(\mathbf{r})\right\rangle,\left|E_{z}(\mathbf{r})\right\rangle\right\}\left(\xi_{12} \rightarrow \pi-\xi_{12}, \xi_{23} \rightarrow \xi_{23}+\pi\right)$, the adiabatic electronic wave vectors $\left|\psi_{1}\right\rangle$ and $\left|\psi_{3}\right\rangle$ will change direction (i.e., the corresponding electronic wave functions change sign). In other words, $\left|\psi_{1}\right\rangle$ and $\left|\psi_{3}\right\rangle$ will be subject to the GP effect while $\left|\psi_{2}\right\rangle$ is not. Of course, state labeling is arbitrary, and hence the conclusion is that only one pair of states changes sign. We may then think of $2 \xi_{12}$ and $2 \xi_{23}$ in the 5-D nuclear configuration space spanned by $\left(Q_{E x}, Q_{E y}, Q_{T x}, Q_{T y}, Q_{T z}\right)$ as being pseudorotational angles for the nuclei. In this case, too, the GP effect is shown to be a property inherent to inversion of the adiabatic electronic wave vectors, now in the space $\left\{\left|E_{x}(\mathbf{r})\right\rangle,\left|E_{y}(\mathbf{r})\right\rangle,\left|E_{z}(\mathbf{r})\right\rangle\right\}$. The above result seems to corroborate the observation made by Chancey and O'Brien [10] that the appearance of a GP in the $T \otimes\left(e+t_{2}\right)$ system is tied to the occurrence of a ground-state triplet. Moreover, it suggests that the procedure adopted for the $N=2$ and 3 Jahn-Teller cases warrants generalization to higher index values.

We may proceed by defining the following real electronic wave functions:

$$
\begin{array}{r}
\psi_{1}=\left(\begin{array}{c}
\cos \gamma_{23} \sin \gamma_{12} \\
\sin \gamma_{23} \sin \gamma_{12} \\
\cos \gamma_{12}
\end{array}\right), \quad \psi_{2}=\left(\begin{array}{c}
\cos \gamma_{23} \cos \gamma_{12} \\
\sin \gamma_{23} \cos \gamma_{12} \\
-\sin \gamma_{12}
\end{array}\right), \\
\psi_{3}=\left(\begin{array}{c}
-\sin \gamma_{23} \\
\cos \gamma_{23} \\
0
\end{array}\right),
\end{array}
$$

where $\gamma_{12}(\mathbf{R})$ is the first mixing angle (which describes mixing of $\psi_{1}$ and $\psi_{2}$ to form an intermediate state $\left.\psi_{12}\right)$ and $\gamma_{23}(\mathbf{R})$ is the second mixing angle (which describes mixing of $\psi_{12}$ and $\psi_{3}$ ). In addition, by analogy with Eq. (20), we write the complex wave function as

$$
\tilde{\psi}=\frac{1}{\sqrt{3}} \sum_{n=1}^{3} \exp \left[\frac{2(n-1) \pi i}{3}\right] \psi_{n}
$$

which, upon evaluation using Eq. (28), yields

$$
\langle\tilde{\psi} \mid \nabla \tilde{\psi}\rangle=-\frac{i}{\sqrt{3}}\left(\nabla \gamma_{12}+\cos \gamma_{12} \nabla \gamma_{23}-\sin \gamma_{12} \nabla \gamma_{23}\right) .
$$

Thus, if we extend Eq. (23) and Eq. (24) to define $A(\mathbf{R})$, we obtain

$$
\langle\tilde{\psi} \mid \nabla \tilde{\psi}\rangle=i \nabla A(\mathbf{R}) .
$$

Comparing Eq. (30) with Eq. (31) then yields

$$
\nabla A(\mathbf{R})=-\frac{1}{\sqrt{3}}\left(\nabla \gamma_{12}+\cos \gamma_{12} \nabla \gamma_{23}-\sin \gamma_{12} \nabla \gamma_{23}\right),
$$

which provides an explicit relation between the GP angle and the mixing angles $\gamma_{12}(\mathbf{R})$ and $\gamma_{23}(\mathbf{R})$. We emphasize that there are three possible mixing angles, from which we may define the two independent Lie group parameters (see also the next subsection).

\subsection{THE $N=4$ CASE: $G \otimes(g+h)$}

Consider now a four-fold electronic degeneracy, which occurs in Jahn-Teller systems such as $(s+p)$ $\otimes\left(a_{1 g}+t_{1 u}+e_{g}+t_{2 g}\right)$ and $G \otimes(g+h)$. The Lie group symmetry of the corresponding Jahn-Teller Hamiltonians with inclusion of linear vibronic interactions [25] is in this case $O(4)$. Thus, there will 
be six Lie group parameters. On the other hand, in the four-fold degeneracy problem, we have 4 electronic wave vectors and hence 6 possible mixing angles (one for each pair). Such electronic wave vectors define a 4-D space in which an arbitrary adiabatic electronic vector can be characterized by its modulus and three angles. The question then arises of how to relate these three angles to the six parameters predicted from Lie group theory (or, equivalently, to the six mixing angles). In other words, the general $\mathbf{T}_{4}$ matrix assumes the form

$$
\mathbf{T}_{4}=\mathbf{t}_{12}\left(\xi_{12}\right) \mathbf{t}_{23}\left(\xi_{23}\right) \mathbf{t}_{34}\left(\xi_{34}\right) \mathbf{t}_{45}\left(\xi_{45}\right) \mathbf{t}_{56}\left(\xi_{56}\right) \mathbf{t}_{61}\left(\xi_{61}\right)
$$

although only three of these $4 \times 4$ unitary planar rotation matrices (one for each mixing angle) can be treated independently. In principle, any set of such angles (out of a total of 20 possibilities) will be equally appropriate, but the three corresponding matrices must form an ordered sequence in order to lead to physically meaningful solutions: upon inversion, an arbitrary wave vector must either change sign or remain sign unchanged. Possible selections are therefore $\mathbf{t}_{12} \mathbf{t}_{23} \mathbf{t}_{34}, \mathbf{t}_{34} \mathbf{t}_{45} \mathbf{t}_{56}$, and so on, but not, e.g., $\mathbf{t}_{12} \mathbf{t}_{45} \mathbf{t}_{61}$. Of course, because all indices are equivalent, any ordered sequence can be relabeled as $\mathbf{T}_{4}=\mathbf{t}_{12} \mathbf{t}_{23} \mathbf{t}_{34}$, which is the one chosen for the following analysis.

Following the previous cases, the real electronic wave functions will now be given by the four rows of $\mathbf{T}_{4}$, namely,

$$
\begin{array}{r}
\psi_{1}=\left(\begin{array}{c}
-S_{12} S_{23} S_{34} \\
S_{12} C_{23} \\
S_{12} S_{23} C_{34} \\
C_{12}
\end{array}\right), \quad \psi_{2}=\left(\begin{array}{c}
-C_{12} S_{23} S_{34} \\
C_{12} C_{23} \\
C_{12} S_{23} C_{34} \\
-S_{12}
\end{array}\right), \\
\psi_{3}=\left(\begin{array}{c}
-C_{23} S_{34} \\
-S_{23} \\
C_{23} C_{34} \\
0
\end{array}\right), \quad \psi_{4}=\left(\begin{array}{c}
-C_{34} \\
0 \\
-S_{34} \\
0
\end{array}\right),
\end{array}
$$

where $C_{i j}=\cos \xi_{i j}$ and $S_{i j}=\sin \xi_{i j}$. We now require a procedure for the inversion operation in four dimensions, $\hat{\imath}_{4}$. As we are not aware of such a procedure, it will be based on requiring that the image point obtained upon application of $\hat{\imath}_{4}$ must show its $n$ coordinates $x_{i}$ sign-reversed. For a vector of unit length in four dimensions, one has

$$
\begin{gathered}
x_{1}=C_{12}, \quad x_{2}=S_{12} C_{23}, \\
x_{3}=S_{12} S_{23} C_{34}, \quad x_{4}=S_{12} S_{23} S_{34},
\end{gathered}
$$

and hence it is easy to show that inversion $\hat{\imath}_{4}$ corresponds to $\xi_{12} \rightarrow \pi-\xi_{12}, \xi_{23} \rightarrow \pi-\xi_{23}$, and $\xi_{34} \rightarrow$ $\pi+\xi_{34}$. Clearly, the wave functions $\psi_{1}$ and $\psi_{4}$ in Eq. (34) are seen to change sign, while the signs of $\psi_{2}$ and $\psi_{3}$ remain unchanged. This corresponds to case $(2,2)$ of Ref. [23] and has no correspondence in the model Hamiltonian work of Ref. [22].

We proceed by calculating the first-derivative coupling terms as a function of the mixing angles. After some algebra, one obtains

$$
\begin{array}{r}
\left\langle\psi_{1} \mid \nabla \psi_{2}\right\rangle=-\nabla \gamma_{12}, \quad\left\langle\psi_{1} \mid \nabla \psi_{3}\right\rangle=-s_{12} \nabla \gamma_{23}, \\
\left\langle\psi_{1} \mid \nabla \psi_{4}\right\rangle=-s_{12} s_{23} \nabla \gamma_{34}, \\
\left\langle\psi_{2} \mid \nabla \psi_{3}\right\rangle=-c_{12} \nabla \gamma_{23}, \quad\left\langle\psi_{2} \mid \nabla \psi_{4}\right\rangle=-c_{12} s_{23} \nabla \gamma_{34}, \\
\left\langle\psi_{3} \mid \nabla \psi_{4}\right\rangle=-c_{23} \nabla \gamma_{34}
\end{array}
$$

where $c_{i j}=\cos \gamma_{i j}$ and $s_{i j}=\sin \gamma_{i j}$. By writing the complex electronic wave function as

$$
\tilde{\psi}=\frac{1}{\sqrt{4}} \sum_{n=1}^{4} \exp \left[\frac{2(n-1) \pi i}{4}\right] \psi_{n}
$$

one now obtains

$$
\begin{aligned}
\langle\tilde{\psi} \mid \nabla \tilde{\psi}\rangle=\frac{i}{\sqrt{4}}\left(\left\langle\psi_{1} \mid \nabla \psi_{2}\right\rangle\right. & +\left\langle\psi_{2} \mid \nabla \psi_{3}\right\rangle \\
& \left.+\left\langle\psi_{3} \mid \nabla \psi_{4}\right\rangle+\left\langle\psi_{4} \mid \nabla \psi_{1}\right\rangle\right),
\end{aligned}
$$

and, by defining the GP angle $A(\mathbf{R})$ as in previous cases, one obtains

$$
\begin{aligned}
\nabla A(\mathbf{R})=-\frac{1}{\sqrt{4}}\left(\nabla \gamma_{12}+\right. & c_{12} \nabla \gamma_{23} \\
& \left.+c_{23} \nabla \gamma_{34}-s_{12} s_{23} \nabla \gamma_{34}\right),
\end{aligned}
$$

which provides a novel explicit relation for the GP angle in terms of the mixing angles.

\subsection{THE ARBITRARY N-INTEGRAL CASE}

Finally, we consider the integral $(N \geq 3)$-fold degeneracy case. The necessary orthogonal transformation matrix assumes the form

$$
\mathbf{T}_{N}=\mathbf{t}_{12}\left(\xi_{12}\right) \mathbf{t}_{23}\left(\xi_{23}\right) \mathbf{t}_{34}\left(\xi_{34}\right) \cdots \mathbf{t}_{N-1, N}\left(\xi_{N-1, N}\right)
$$

where $\mathbf{t}_{n m}$ are $N \times N$ matrices defined in a manner similar to Eq. (33). The $N$ real electronic wave func- 
tions now become the $N$ rows of $\mathbf{T}_{N}$, which can be written by induction using the previous cases. To examine the effect of the inversion $\hat{\imath}_{N}$, we then require the following generalization of Eq. (35):

$$
\begin{gathered}
x_{1}=C_{12} \\
x_{k}=\left(\prod_{i=1}^{k-1} S_{i, i+1}\right) C_{k, k+1} \quad(k=2,3, \ldots, N-1) \\
x_{N}=\left(\prod_{i=1}^{N-1} S_{i, i+1}\right)
\end{gathered}
$$

As seen by induction from previous cases, $\hat{\imath}_{N}$ implies $\gamma_{i, i+1} \rightarrow \pi-\gamma_{i, i+1}(i=1,2, \ldots, N-2)$, and $\gamma_{N-1, N} \rightarrow \pi+\gamma_{N-1, N}$.

After extensive algebraic manipulation, one obtains for $\left\langle\psi_{k}^{(N)} \mid \nabla \psi_{i}^{(N)}\right\rangle(i=1,2, N)$

$$
\begin{array}{r}
\left\langle\psi_{k}^{(N)} \mid \nabla \psi_{1}^{(N)}\right\rangle=\delta_{2, k} \nabla \gamma_{12} \\
+\sum_{m=1}^{N-2} \prod_{j=1}^{m} s_{j, j+1} \delta_{m+2, k} \nabla \gamma_{m+1, m+2} \\
\left\langle\psi_{k}^{(N)} \mid \nabla \psi_{2}^{(N)}\right\rangle=-\delta_{1, k} \nabla \gamma_{12}+c_{12} \delta_{3, k} \nabla \gamma_{23} \\
+c_{12} \sum_{m=2}^{N-2} \prod_{i=2}^{m} s_{i, i+1} \delta_{m+2, k} \nabla \gamma_{m+1, m+2} \\
\left\langle\psi_{k}^{(N)} \mid \nabla \psi_{N}^{(N)}\right\rangle=\left\langle\psi_{k}^{(N)} \mid \frac{\partial \psi_{N}^{(N)}}{\partial \gamma_{N-1, N}}\right\rangle \nabla \gamma_{N-1, N}
\end{array}
$$

In turn, for $i=N-m \geq 3$, one obtains

$$
\begin{gathered}
\left.\left\langle\psi_{k}^{(N)} \mid \nabla \psi_{N-m}^{(N)}\right\rangle=\left\langle\psi_{k}^{(N)}\right| \frac{\partial \psi_{N-m}^{(N)}}{\partial \gamma_{N-m-1, N-m}}\right) \nabla \gamma_{N-m-1, N-m} \\
\quad+c_{N-m-1, N-m}\left(\delta_{N-m+1, k} \nabla \gamma_{N-m, N-m+1}\right. \\
\left.+\sum_{l=0}^{N-5} \prod_{j=0}^{l} s_{N-m+j, N-m+j+1} \delta_{N-m+2+l, k} \nabla \gamma_{N-m+1+l, N-m+2+l}\right) .
\end{gathered}
$$

Note that the only nonzero integrals $\left\langle\psi_{k}^{(N)}\right|\left(\partial \psi_{N}^{(N)} /\right.$ $\left.\left.\partial \gamma_{i j}\right)\right\rangle$ in Eq. (43) and Eq. (44) are

$$
\begin{gathered}
\left\langle\psi_{1} \mid \frac{\partial \psi_{3}}{\partial \gamma_{23}}\right\rangle=-s_{12}, \quad\left\langle\psi_{2} \mid \frac{\partial \psi_{3}}{\partial \gamma_{23}}\right\rangle=-c_{12}, \\
\left\langle\psi_{1} \mid \frac{\partial \psi_{4}}{\partial \gamma_{34}}\right\rangle=-s_{12} s_{23}, \quad\left\langle\psi_{2} \mid \frac{\partial \psi_{4}}{\partial \gamma_{34}}\right\rangle \\
=-c_{12} s_{23}, \quad\left\langle\psi_{3} \mid \frac{\partial \psi_{4}}{\partial \gamma_{34}}\right\rangle=-c_{23}, \\
\left\langle\psi_{1} \mid \frac{\partial \psi_{5}}{\partial \gamma_{45}}\right\rangle=-s_{12} s_{23} s_{34}, \quad\left\langle\psi_{2} \mid \frac{\partial \psi_{5}}{\partial \gamma_{45}}\right\rangle=-c_{12} s_{23} s_{34},
\end{gathered}
$$

$$
\left\langle\psi_{3} \mid \frac{\partial \psi_{5}}{\partial \gamma_{45}}\right\rangle=-c_{23} s_{34}, \quad\left\langle\psi_{4} \mid \frac{\partial \psi_{5}}{\partial \gamma_{45}}\right\rangle=-c_{34} .
$$

By now writing the $\mathrm{N}$-state complex electronic wave function as

$$
\tilde{\psi}=\frac{1}{\sqrt{N}} \sum_{n=1}^{N} \exp \left[\frac{2(n-1) \pi i}{N}\right] \psi_{n}
$$

we obtain for the derivative coupling term

$$
\langle\tilde{\psi} \mid \nabla \tilde{\psi}\rangle=\frac{1}{N} \sum_{m \neq n}^{N} \exp \left[\frac{2(n-m) \pi i}{N}\right]\left\langle\psi_{m} \mid \nabla \psi_{n}\right\rangle
$$

or

$$
\begin{array}{r}
\langle\tilde{\psi} \mid \nabla \tilde{\psi}\rangle=\frac{i}{\sqrt{N}}\left(\left\langle\psi_{1} \mid \nabla \psi_{2}\right\rangle+\left\langle\psi_{2} \mid \nabla \psi_{3}\right\rangle+\left\langle\psi_{3} \mid \nabla \psi_{4}\right\rangle\right. \\
\left.+\cdots+\left\langle\psi_{N} \mid \nabla \psi_{1}\right\rangle\right) .
\end{array}
$$

If $A(\mathbf{R})$ is then defined similarly to previous cases, one obtains

$$
\begin{aligned}
\nabla A(\mathbf{R})=\frac{1}{\sqrt{N}}\left(\left\langle\psi_{1} \mid \nabla \psi_{2}\right\rangle+\left\langle\psi_{2} \mid \nabla \psi_{3}\right\rangle\right. & \\
& \left.+\left\langle\psi_{3} \mid \nabla \psi_{4}\right\rangle+\cdots+\left\langle\psi_{N} \mid \nabla \psi_{1}\right\rangle\right),
\end{aligned}
$$

which gives the explicit relation between the GP angle and the first derivative coupling terms. Moreover, by using Eq. (43) and Eq. (44), we obtain the relation between the GP angle and the $N-1$ mixing angles for the general $N \geq 3$ state case: 


$$
\begin{gathered}
\nabla A(\mathbf{R})=-\frac{1}{\sqrt{N}}\left(\nabla \gamma_{12}+\sum_{n=1}^{N-2} c_{n, n+1} \nabla \gamma_{n+1, n+2}\right. \\
\left.\quad-\prod_{n=1}^{N-2} s_{n, n+1} \nabla \gamma_{N-1, N}\right) .
\end{gathered}
$$

\section{Conclusions}

We now summarize the major results. For an $N$-fold electronic degeneracy in Jahn-Teller systems, propagation of the adiabatic electronic wave vectors around the point of degeneracy can be represented as a rotation in $N-1$ parameters in the $N$-dimensional electronic wave-vector space. Inversion in such a space leads to the GP effect. Thus, for $N=2$, any adiabatic electronic wave vector can be represented as a one-parameter (i.e., the mixing angle, which is identical up to an initial phase to the GP angle [16, 17]) rotation in the 2-D wave-vector space. For $N=3$, such a rotation will take place in the 3-D electronic wave-vector space, with inversion then showing that only two adiabatic electronic wave vectors are subject to the GP effect. Correspondingly, the $N=4$ case can be viewed by looking at the action of $\hat{\imath}_{4}$. The result is that two electronic wave vectors change sign and hence are subject to the GP effect, while the other two are not. For an arbitrary integer $-N$ value, such a rotation involves $N-1$ mixing angles as parameters in the $N$-dimensional electronic wave-vector space. To look at the GP effect, we must then examine the results of applying $\hat{\imath}_{N}$ to the adiabatic electronic wave vectors. As already observed, we are not expected to obtain all sign changes reported by Manolopoulos and Child [22] and Baer [23], since only Jahn-Teller systems with very specific symmetry requirements have been considered in this work. Finally, the GP angle for the general $N$-state case has been shown for the first time to connect the $N-1$ independent mixing angles.

\section{ACKNOWLEDGMENT}

This was has been supported by the Fundaçáo para a Ciência e Tecnologia, Portugal.

\section{References}

1. Herzberg, G.; Longuet-Higgins, H. C. Faraday Discuss Chem Soc 1963, 35, 77.

2. Mead, C. A. Rev Mod Phys 1992, 64, 51.

3. Yarkony, D. R. Rev Mod Phys 1996, 68, 985.

4. Varandas, A. J. C.; Xu, Z. R. In Advances in Chemistry and Physics; Baer, M.; Billing, G. D., Eds.; Wiley: New York; 2002; p 659.

5. Berry, M. V. Proc R Soc 1984, A392, 45.

6. Mead, C. A. Chem Phys 1980, 49, 23.

7. Mead, C. A.; Truhlar, D. G. J Chem Phys 1979, 70, 2284.

8. Mead, C. A. J Chem Phys 1980, 72, 3839.

9. Ham, F. S. Phys Rev Lett 1987, 58, 725.

10. Chancey, C. C.; O’Brien, M. C. M. J Phys A: Math Gen 1988, $21,3347$.

11. Varandas, A. J. C.; Yu, H. G. J Chem Soc Faraday Trans 1997, 93, 819.

12. Baer, M.; Englman, R. Chem Phys Lett 1997, 265, 105.

13. Baer, M. J Chem Phys 1997, 107, 2694.

14. Kendrick, B. K.; Mead, C. A.; Truhlar, D. G. J Chem Phys 1999, 110, 7594.

15. Varandas, A. J. C.; Xu, Z. R. Chem Phys Lett 2000, 316, 248.

16. Varandas, A. J. C.; Xu, Z. R. J Chem Phys 2000, 112, 2121.

17. Xu, Z. R.; Baer, M.; Varandas, A. J. C. J Chem Phys 2000, 112, 2746.

18. Xu, Z. R.; Varandas, A. J. C. Int J Quantum Chem 2000, 80, 454.

19. Xu, Z. R.; Varandas, A. J. C. Int J Quantum Chem 2001, 83, 279.

20. Xu, Z. R.; Varandas, A. J. C. J Phys Chem 2001, 105, 2246.

21. Kendrick, B.; Mead, C. A.; Truhlar, D. G. Chem Phys Lett 2002, 277, 31.

22. Manolopoulos, D. E.; Child, M. S. Phys Rev Lett 1999, 82, 2223.

23. Baer, M. Chem Phys Lett 2000, 322, 520.

24. Englman, R. The Jahn-Teller Effect; Wiley: New York; 1973.

25. Bersuker, I. B.; Polinger, V. Z. Vibronic Interactions in Molecules and Crystals; Springer-Verlag: Berlin, 1989.

26. Chrisholm, C. D. H. Group Theoretical Techniques in Quantum Chemistry; Academic Press: San Diego, 1976.

27. Hammermesh, M. Group Theory and Its Application to Physical Problems; Dover: New York, 1989.

28. Leung, C. H.; Kleiner, W. H. Phys Rev B 1974, 10, 4434.

29. Varandas, A. J. C. J Chem Phys 1997, 107, 867.

30. Baer, M. Chem Phys 1976, 15, 49.

31. Baer, M. Mol Phys 1980, 40, 1011.

32. Pacher, T.; Cederbaum, L. S.; Köppel, H. Adv Chem Phys 1993, 84, 293.

33. Kryachko, E. S.; Varandas, A. J. C. Int J Quantum Chem 2002, 89, 255.

34. Baer, M. J Chem Phys 1997, 107, 10662.

35. Leon, S. J. Linear Algebra with Applications; MacMillan Publishing Company: New York, 1990. 